\title{
Differential Evolution Algorithm Aided Minimum Symbol Error Rate Multi-user Detection for Multi-user OFDM/SDMA Systems
}

\author{
Jiankang Zhang ${ }^{\dagger *}$, Sheng Chen*, Xiaomin $\mathrm{Mu}^{\dagger}$, Lajos Hanzo* \\ $\uparrow$ School of Information Engineering, Zhengzhou University, Zhengzhou, China, \\ * School of ECS, University of Southampton, SO17 1BJ, United Kingdom. \\ Tel: +44-23-8059 3125, Fax: +44-23-8059 4508 \\ Email: jz09v@ecs.soton.ac.uk,sqc@ecs.soton.ac.uk, iexmmu@zzu.edu.cn,1h@ecs.soton.ac.uk \\ http://www-mobile.ecs.soton.ac.uk
}

\begin{abstract}
A Differential Evolution (DE) algorithm assisted Minimum Symbol Error Ratio (MSER) Multi-User Detection (MUD) scheme is proposed for multi-user Multiple-Input Multiple-Output (MIMO) aided Orthogonal Frequency-Division Multiplexing / Space Division Multiple Access (OFDM/SDMA) systems. Quadrature Amplitude Modulation (QAM) is employed in most wireless standards by virtue of providing a high throughput. The MSER Cost Function (CF) may be deemed to be the most relevant one for QAM, but finding its minimum is challenging. Hence we propose a sophisticated DE assisted MSER-MUD scheme, which directly minimizes the SER CF of multi-user OFDM/SDMA systems employing QAM. Furthermore, the effects of the DE assisted MSER-MUD's algorithmic parameters, namely those of the population size $P_{s}$, of the scaling factor $\lambda$ and of the crossover probability $C_{r}$ on the number of $\mathrm{DE}$ generations required for attaining convergence were investigated in our simulations. This allowed us to directly quantify their complexity. The simulation results also demonstrate that the proposed DE assisted MSER-MUD scheme significantly outperforms the conventional MMSE-MUD in term of the system's overall BER and it is capable of narrowing its BER performance discrepancy with respect to the optimal Maximum Likelihood (ML) MUD to about $4 d B$, while requiring about 200 times less CF evaluations compared to the optimal ML-MUD scheme.
\end{abstract}

Index Terms-Orthogonal frequency division multiplexing (OFDM), space division multiple access (SDMA), minimum symbol error rate, multi-user detection, differential evolution algorithm.

\section{INTRODUCTION}

The best possible exploitation of the finite available spectrum in the light of the increasing demands for wireless services has been at the centre of wireless system optimization. Multiple antennas can be employed both at the transmitter and/or the receiver, which leads to the concept of Multiple Input Multiple Output (MIMO) systems, in order to attain improvements in both capacity and Bit Error Rates (BER) [1,2]. As one of the most wide-spread MIMO types, Spatial Division Multiple Access/Orthogonal Frequency-Division Multiplexing (OFDM/SDMA) systems [3] exploit the advantages of both OFDM and SDMA, which increase the systems' capacity by sharing the same bandwidth and time slots by several users roaming in different geographical locations [1].

More specifically, the transmitted signals of $U$ simultaneous singleantenna aided UpLink (UL) Mobile Stations (MSs) are received by an array of antennas at the Base Station (BS). At the BS, Multi-User Detection (MUD) techniques are invoked for separating the signals of the different MSs. Over the past decade, a variety of SDMA MUDs have been proposed for separating the users' data on the basis of their unique, user-specific 'spatial signature', i.e. the Channel Impulse Responses (CIRs). Naturally, for near-single-user MUD the CIRs have to be accurately estimated [1]. As one of the most popular linear SDMA-receiver design strategy, Minimum Mean Square Error (MMSE) MUD [3] strikes a tradeoff between the achievable MultiUser Interference (MUI) rejection and noise amplification. In [4], a

Acknowledgments: The financial support of the China Scholarship Council (CSC) and the EPSRC under the auspices of the China-UK Science Bridge as well as of the RC-UK under the India-UK Advanced Technology centre initiative is gratefully acknowledged.
Constrained Least Squares (CLS) detector was designed for constant modulus signals, which exploited the constant modulus nature of the subcarrier modulation. This CLS-MUD outperformed the MMSE MUD, despite its lower computational complexity. In general, the MMSE-MUD [3] and the CLS-MUD [4] may be viewed as linear MUDs, which minimize the Mean Square Error (MSE) at the MUD's output. However, it is not the MSE, but the BER or Symbol Error Ratio (SER) that really matters in most communications system. This is, because minimizing the MSE does not necessarily guarantee that the BER or SER of the communication system is also minimized, unless the MUD's output signal is strictly Gaussian [5]. This has motivated the quest for directly minimizing the system's BER or SER.

Over the past decade, intensive research efforts have been devoted to the formulation and exploitation of the Minimum BER (MBER) criterion in diverse applications [6], including MUDs in both Codedivision Multiple-Access (CDMA) systems [7] and space-time equalized SDMA systems [5, 8,9]. Since, Quadrature Amplitude Modulation (QAM) schemes have become popular in wireless standards by virtue of providing a high throughput, direct Minimum SER (MSER) detection was conceived for $M$-QAM systems [10].

The MUD schemes based on the MBER criterion $[5,8,9]$ have paved the way for conceiving a novel Differential Evolution (DE) $[11,12]$ assisted MSER-MUD. However, the extension of the MBERMUD originally designed for Binary Phase Shift Keying (BPSK) assisted systems to the MSER-MUD is non-trivial, as demonstrated in this paper. Against this background, our new contribution is the formulation of the MSER criterion for MUD in OFDM/SDMA systems and its solution with the aid of DE optimization. More specifically, we study the effects of the DE-aided MSER-MUD's algorithmic parameters, namely those of the population size $P_{s}$, of the scaling factor $\lambda$ and of the crossover probability $C_{r}$, which allows us to characterize the attainable performance versus the complexity imposed.

The rest of this paper is organized as follows. The system model of the multi-user OFDM/SDMA UL is described in Section II. Section III is devoted to formulating the MSER solution for the MUD of the multi-user OFDM/SDMA systems. In Section IV, we will illustrate the proposed DE assisted MSER-MUD. Our simulation results and discussions are presented in Section V, while our conclusions are offered in Section VI.

\section{System MODEL}

The multi-user OFDM/SDMA UL system considered is shown in Fig. 1, where each of the $U$ simultaneous users is equipped with a single transmission antenna, while the BS employs an array of $Q$ antennas. All users simultaneously transmit their independent data streams, denoted by $\mathbf{b}^{u}, u=1,2, \cdots, U$. The information bits $\mathbf{b}^{u}$ are first encoded by the independent Low-Density ParityCheck (LDPC) encoder of each user, as seen in Fig. 1. The data stream is modulated and then the pilot symbols are embedded into the Frequency Domain (FD) representation of each OFDM symbol. 


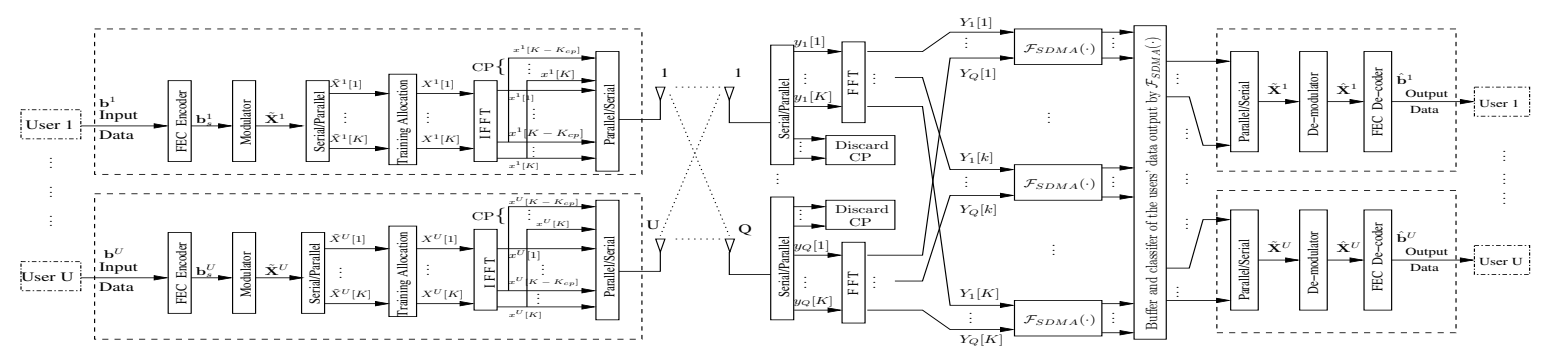

Fig. 1. Uplink system model for Multi-user MIMO OFDM/SDMA.

These FD pilot symbols and their specific allocation are known at the receiver and hence can be exploited for channel estimation. Then the signals are fed to a classic $K$-point Inverse Fast Fourier Transform (IFFT) based modulator in order to generate the Time-Domain (TD) modulated signal. After concatenating the Cyclic-Prefix (CP) of $K_{c p}$ samples, the resultant sequence is transmitted through the MIMO channel.

At the BS, the received signals $\mathbf{y}_{q}$ of antenna $q, q=1,2, \cdots, Q$, are constituted by the superposition of the independently faded TD signals of the $U$ users sharing the same space-frequency resource, which are also corrupted by the Gaussian noise at the array elements. After discarding the $\mathrm{CP}$ and performing FFT-based demodulation of the received TD signals, we generate $Q$ separate received sequences for the $s$-th OFDM symbol $\mathbf{Y}_{q}[s], q=1,2, \cdots, Q$, which is given by the superposition of the different users' channel-impaired received signal contribution plus the Additive White Gaussian Noise (AWGN), formulated as:

$$
\mathbf{Y}_{q}[s]=\sum_{u=1}^{U} \mathbf{X}^{u}[s] \mathbf{H}_{q}^{u}[s]+\mathbf{n}_{q}[s],
$$

where $\mathbf{Y}_{q}[s] \in \mathbb{C}^{K \times 1}, \mathbf{H}_{q}^{u}[s] \in \mathbb{C}^{K \times 1}$ and $\mathbf{n}_{q}[s] \in \mathbb{C}^{K \times 1}$ in Equation (1) are column vectors hosting the subcarrier-related received signal $Y_{q}[s, k]$, the Frequency Domain Channel Transfer Factors (FD-CHTFs) $H_{q}^{u}[s, k]$ and the AWGN $n_{q}[s, k]$, respectively. Furthermore, $\mathbf{X}^{u}[s] \in \mathbb{C}^{K \times K}$ is a diagonal matrix with elements given by $X^{u}[s, k], k=1,2, \cdots, K$ which represents the $U$ users' transmitted signals, assuming values from the $M$-QAM symbol set of

$$
\mathcal{S} \triangleq\left\{s_{m, n} \mid s_{m, n}=z_{m}+j z_{n}, 1 \leq m, n \leq \sqrt{M}\right\},
$$

where the real-part of the symbols is $\Re\left\{s_{m, n}\right\}=z_{m}=2 m-\sqrt{M}-$ 1 and the imaginary-part is $\mathfrak{I}\left\{s_{m, n}\right\}=z_{n}=2 n-\sqrt{M}-1$.

\section{The MSER-BASED MUD SCHEME}

Upon invoking vector notations, the set of equations constituted by Equation (1) for $q=1,2, \cdots, Q$ at the $k$-th subcarrier of the $s$-th OFDM symbol can be rewritten as:

$$
\mathbf{Y}[s, k]=\mathbf{H}[s, k] \mathbf{X}[s, k]+\mathbf{n}[s, k],
$$

where $\mathbf{Y}[s, k] \in \mathbb{C}^{Q \times 1}, \mathbf{H}[s, k] \in \mathbb{C}^{Q \times U}, \mathbf{X}[s, k] \in \mathbb{C}^{U \times 1}$ and $\mathbf{n}[s, k] \in \mathbb{C}^{Q \times 1}$ represent the received signals, the FD MIMO channel matrix, the transmitted signals and the AWGN noise, respectively. For notational convenience, the indices $[s, k]$ are omitted during our forthcoming discourse. The estimate $\hat{\mathbf{X}}$ of the transmitted signal vector $\mathbf{X}$ of the $U$ simultaneous users can be generated by the MUD upon linearly combining the signals received by the $Q$ different antennas at the BS with the aid of the array weight matrix $\mathbf{W}$, yielding $[1,5]$,

$$
\tilde{\mathbf{X}}=\mathbf{W}^{H} \overline{\mathbf{Y}}+\mathbf{W}^{H} \mathbf{n},
$$

where the superscript of $(\cdot)^{H}$ denotes the Hermitian transpose and $\overline{\mathbf{Y}}=\mathbf{H X}$ represents the noise-free received data. Since the transmitted signals of different users are independent from each other, the signals output by the MUD can be on a per user basis. Considering the $u$-th users' associated signal, we arrive at

$$
\tilde{X}^{u}=\bar{X}^{u}+e^{u},
$$

where $e^{u}=\mathbf{W}^{u H} \mathbf{n}^{1}$ represents the noise with zero mean and a variance of $\sigma_{n}^{2} \mathbf{W}^{u H} \mathbf{W}^{u}$, while $\bar{X}^{u}=\mathbf{W}^{u H} \overline{\mathbf{Y}}^{u}$ denotes the noisefree signal output by the MUD.

The Probability Density Function (PDF) of the real-part $\tilde{X}_{R}^{u}$ of $\tilde{X}^{u}$ conditioned both on $X^{u}=z_{m}$ and on $\mathbf{W}^{u}$ is a Gaussian mixture, which may be readily formulated as [13]

$f\left(\tilde{X}_{R}^{u} \mid \bar{X}_{R \mid X_{R}^{u}=z_{m}}^{u}, \mathbf{W}^{u}\right)=\frac{1}{\sqrt{M}} \sum_{n=1}^{\sqrt{M}} f\left(\tilde{X}_{R}^{u} \mid \bar{X}_{R \mid X_{R}^{u}=z_{m}+j z_{n}}^{u}, \mathbf{W}^{u}\right)$

where $f\left(\tilde{X}_{R}^{u} \mid \bar{X}_{R \mid X_{R}^{u}=z_{m}+j z_{n}}^{u}, \mathbf{W}^{u}\right)$ represents the PDF of $\tilde{X}_{R}^{u}$ conditioned both on $X^{u}=z_{m}+j z_{n}$ and on $\mathbf{W}^{u}$, while $\bar{X}_{R \mid X_{R}^{u}=z_{m}+j z_{n}}^{u}$ is constituted by those $N_{s b}=M^{U-1}$ specific trial vectors, whose $u$-th elements has a value of $\left(z_{m}+j z_{n}\right)$.

Given that $\left(z_{1}+j z_{1}\right)$ was transmitted by user- $u$, the probability of error for the real-part $\tilde{X}_{R}^{u}$ is simply the probability that we have $\tilde{X}_{R}^{u}<z_{1}+1$, i.e.,

$$
\begin{aligned}
\left.P_{E, u, R}\left(\mathbf{W}^{u}\right)\right|_{X_{R}^{u}=z_{1}} & =\int_{z_{1}+1}^{+\infty} f\left(\tilde{X}_{R}^{u} \mid \bar{X}_{R \mid X_{R}^{u}=z_{1}}^{u}, \mathbf{W}^{u}\right) d \tilde{X}_{R}^{u} \\
& =\frac{1}{2 N_{s b}} \sum_{i=1}^{N_{s b}} \operatorname{erfc}\left[C_{R, i}\left(\mathbf{W}^{u}\right)\right]
\end{aligned}
$$

where $\operatorname{erfc}(\cdot)$ denotes the complementary error function [13] and $C_{R, i}\left(\mathbf{W}^{u}\right)$ is formulated as

$$
C_{R, i}\left(\mathbf{W}^{u}\right)=\frac{\left(z_{1}+1\right)-\bar{X}_{R, i \mid X_{R}^{u}=z_{1}}^{u}}{\sigma_{n} \sqrt{\mathbf{W}^{u H} \mathbf{W}^{u}}} .
$$

Due to symmetry [13], the error probabilities are identical for $X_{R}^{u}=z_{1}$ and $X_{R}^{u}=z_{\sqrt{M}}$, while the error probabilities for $X_{R}^{u}=z_{m}, m=2,3, \cdots, \sqrt{M}-1$ are twice that of $X_{R}^{u}=z_{1}$. Since all the legitimate $M$-QAM signals are equally likely to be transmitted, the total error probability of the real-part $\tilde{X}_{R}^{u}$ becomes

$$
P_{E, u, R}\left(\mathbf{W}^{u}\right)=\frac{\sqrt{M}-1}{\sqrt{M} N_{s b}} \sum_{i=1}^{N_{s b}} \operatorname{erfc}\left[C_{R, i}\left(\mathbf{W}^{u}\right)\right] .
$$

A direct evaluation of Equation (9) imposes a high computational complexity, due to the integration operation in the function $\operatorname{erfc}(\cdot)$. However fortunately the computational complexity of $\operatorname{erfc}(\cdot)$ may be reduced by exploiting that [14]:

$$
\overline{\operatorname{erfc}}(x)= \begin{cases}1+\left[1-e^{-\frac{x^{2}\left(4 / \pi+0.14 x^{2}\right)}{1+0.14 x^{2}}}\right]^{1 / 2}, & x<0, \\ 1-\left[1-e^{-\frac{x^{2}\left(4 / \pi+0.14 x^{2}\right)}{1+0.14 x^{2}}}\right]^{1 / 2}, & x \geq 0 .\end{cases}
$$

The lines marked in Fig. 2 by the dots and circles represent the true values of $\operatorname{erfc}(x)$ and the approximated values of $\overline{\operatorname{erfc}}(x)$,

\footnotetext{
${ }^{1}$ The weight vector $\mathbf{W}^{u}$ is rotated as $\mathbf{W}_{n e w}^{u}=\frac{\mathbf{W}^{u H} \mathbf{H}}{\left\|\mathbf{W}^{u H} \mathbf{H}\right\|} \mathbf{W}^{u}$ and normalized as $\tilde{\mathbf{W}}_{\text {new }}^{u}=\frac{\mathbf{W}_{n e w}^{u}}{\mathbf{W}_{\text {new }}^{u} \mathbf{H}}$
} 


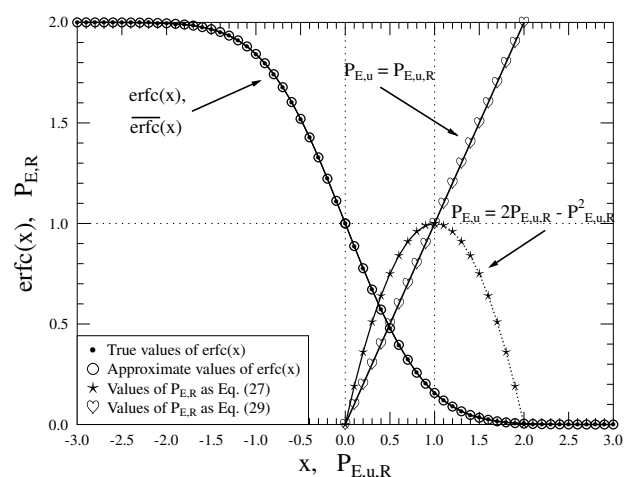

Fig. 2. The chart of the true values of $\operatorname{erfc}(x)$, approximated function of $\operatorname{erfc}(x)$, i.e., $\overline{\operatorname{erfc}}(x)$, the total BER $P_{E, u}$ which regards $P_{E, u, R}$ as a variable and a loose approximation for $P_{E, u}$ as Equation (13).

respectively. It can be seen that the approximate $\overline{\operatorname{erfc}}(x)$ formula of Equation (10) perfectly matches the function $\operatorname{erfc}(x)$.

Assuming that the square-shaped $M$-QAM constellation is considered, the real-part and imaginary-part will be symmetric to each other. Hence, the error probability of the imaginary-part is identical to that of the real-part. Hence the total SER is given by

$$
P_{E, u}\left(\mathbf{W}^{u}\right)=2 P_{E, u, R}\left(\mathbf{W}^{u}\right)-P_{E, u, R}^{2}\left(\mathbf{W}^{u}\right) .
$$

The MSER solution $\mathbf{W}_{M S E R}^{u}$ is defined as the weight vector that minimizes the SER of $P_{E, u}$, which is formulated as

$$
\hat{\mathbf{W}}_{M S E R}^{u}=\arg \min _{\mathbf{W}^{u}} P_{E, u}\left(\mathbf{W}^{u}\right) .
$$

Naturally, we have $0 \leq P_{E, u, R}\left(\mathbf{W}^{u}\right) \leq 1$ in realistic communications systems. However, the initial values of the weight vector $\mathbf{W}^{u}$ are randomly generated in our DE assisted MSER-MUD, which will be elaborated on in the following section. Hence $C_{R, i}\left(\mathbf{W}^{u}\right)$ may become negative, which will result in $\operatorname{erfc}\left[C_{R, i}\left(\mathbf{W}^{u}\right)\right]>1$ and this in turn may result in $P_{E, u, R}\left(\mathbf{W}^{u}\right)>1$. Intuitively, these are flawed solution vectors. However, we can see from Fig. 2 that the total SER $P_{E, u}\left(\mathbf{W}^{u}\right)$ decays beyond the abscissa value of $P_{E, u, R}\left(\mathbf{W}^{u}\right)>1$, which may misguide the MSER-CF-based DE-aided optimization. Hence we set the total SER of $P_{E, u}\left(\mathbf{W}^{u}\right)$ to $^{2}$ :

$$
P_{E, u}\left(\mathbf{W}^{u}\right)=P_{E, u, R}\left(\mathbf{W}^{u}\right) .
$$

\section{Differential Evolution Algorithm Aided MSER-MUD}

Due to the irregular shape of the CF of Equation (11), the MSER solution does not have a closed-form. However, DE optimization algorithms may be employed for assisting the MSER-MUD in finding the minimum of the CF. As a relatively new member in the family of Evolutionary Algorithms (EAs), the DE [11,12] algorithm also constitutes a random guided population-based search method, which employs repeated cycles of candidate-solution re-combination and selection operations for guiding the population towards the vicinity of the global optimum. In this treatise, we employed the DE algorithm to assist the MSER-MUD as a benefit of its versatility in solving these sophisticated optimization problems.

The DE algorithm may be characterized with the aid of its initialization, mutation, re-combination and selection operations invoked for exploring the search space in an iterative progression, until the termination criterion is met. The structure of the proposed DE aided MSER-MUD can be best understood with the aid of the flowchart shown in Fig. 3, which will be often referred to during our forthcoming discourse.

${ }^{2}$ This simplified mathematical expression augments the role of the 'meritorious' candidate vectors and sidelines the flawed solution vectors, although it does not accurately portray the true values of $P_{E, u}\left(\mathbf{W}^{u}\right)$, which are shown in Fig. 2 by the curve marked by hearts.

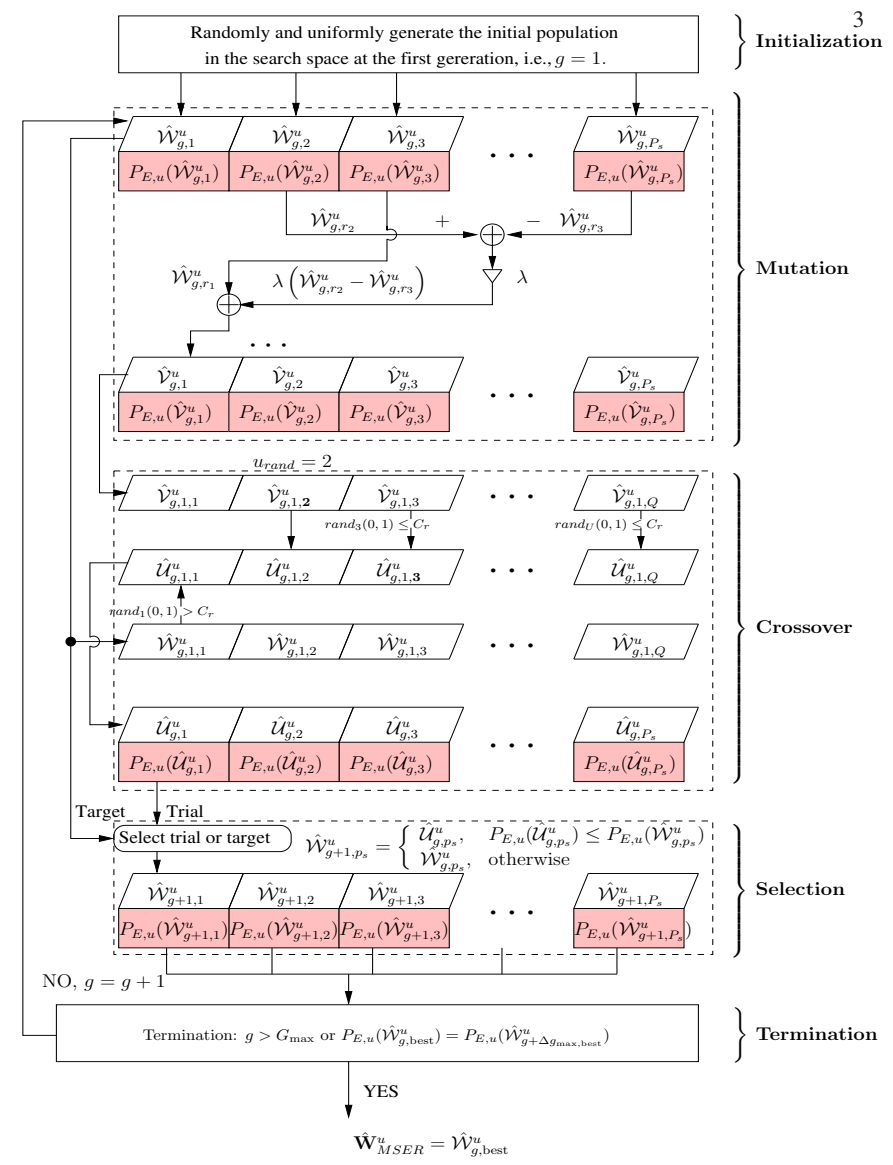

Fig. 3. Flowchart of the differential evolution algorithm.

1) Initialization. $\mathrm{DE}$ algorithm commences its search from a population of $P_{s} Q$-dimensional complex-valued solution vectors. The $p_{s}$-th vector of the population in the first generation of $g=1$ may be readily expressed as

$$
\hat{\mathcal{W}}_{1, p_{s}}^{u}=\left[\hat{\mathcal{W}}_{1, p_{s}, 1}^{u}, \hat{\mathcal{W}}_{1, p_{s}, 2}^{u}, \cdots, \hat{\mathcal{W}}_{1, p_{s}, Q}^{u}\right]^{T},
$$

where again, $u$ is the user-index and $Q$ is the number of antennas.

2) Mutation. The mutation operation allows DE to maintain the diversity of the population, while insightfully steering the optimization. The appropriate choice of the mutation parameters allows DE to prevent 'early convergence' to a local minimum without thoroughly exploring the entire solution space. More specifically, mutation is one of the distinctive features of the DE algorithm, which does not use a predefined probability density function for generating the perturbed solutions. Instead, it relies upon the population itself in perturbing the candidate solutions by adding an appropriately scaled and randomly selected difference-vector ${ }^{3}$ to a base population vector. As seen in Fig. 3, the vector difference is scaled by a problem-specific, optimized scaling factor $\lambda$ invoked for combining two randomly selected population vectors. More specifically, the following equation shows how to create a mutant vector by combining three different, randomly chosen vectors according to

$$
\hat{\mathcal{V}}_{g, i}^{u}=\hat{\mathcal{W}}_{g, r_{1}}^{u}+\lambda\left(\hat{\mathcal{W}}_{g, r_{2}}^{u}-\hat{\mathcal{W}}_{g, r_{3}}^{u}\right)
$$

where the scaling factor $\lambda \in(0,1]$ is a positive real-valued number that controls the rate at which the population evolves and $u$ is the user-index.

3) Crossover. The crossover operation generates a trial vector by replacing certain parameters of the target vector with the corresponding parameters of a randomly selected donor vector. As a significant

\footnotetext{
${ }^{3}$ The difference of the vectors $\hat{\mathcal{W}}_{g, r_{2}}^{u}$ and $\hat{\mathcal{W}}_{g, r_{3}}^{u}$ was defined as $\Delta_{\hat{\mathcal{W}}_{g, r_{2}}^{u}, \hat{\mathcal{W}}_{g, r_{3}}^{u}}=\hat{\mathcal{W}}_{g, r_{2}}^{u}-\hat{\mathcal{W}}_{g, r_{3}}^{u}$, as also seen in Fig. 3
} 
complementarity to the above-mentioned differential mutation, the crossover operation increases the potential diversity of the population vectors. There exist diverse variants of the crossover mechanisms [11, 12]. We opted for employing the uniform crossover algorithm, where each DE parameter, regardless of its location in the trial vector, has the same probability of inheriting its value from a given vector. More particularly, the $q$-th value of the $i$-th vector in the population at the $g$-th generation, namely $\hat{\mathcal{U}}_{g, i, q}^{u}$, is given by

$$
\hat{\mathcal{U}}_{g, i, q}^{u}= \begin{cases}\hat{\mathcal{V}}_{g, i, q}^{u}, & \operatorname{rand}_{q}(0,1) \leq C_{r} \text { or } q=q_{\text {rand }}, \\ \hat{\mathcal{W}}_{g, i, q}^{u}, & \text { otherwise, }\end{cases}
$$

where $C_{r} \in[0,1]$ represents the crossover probability, which is a problem-specific value that represents the specific weight applied to the parameter values that are copied from a previous vector to the mutant, as seen in Fig. 3. Furthermore, $\operatorname{rand}_{q}(0,1)$ denotes a random number generator, which returns a uniformly distributed random value from the range $[0,1)$. The antenna-index $q=1,2, \cdots, Q$ indicates that a new random value is generated for each of the $q$ parameters of user $u=1,2, \cdots, U$.

4) Selection. In order to keep the population size constant over subsequent generations, the selection operator determines whether the target vector $\hat{\mathcal{W}}_{g, p_{s}}^{u}$ or the trial vector $\hat{\mathcal{U}}_{g, p_{s}}^{u}$ survives to the next generation, as seen in Fig. 3. The DE algorithm does not use fitness-based selection for the next generation. Instead, the CF of the trial vector $\hat{\mathcal{U}}_{g, p_{s}}^{u}$, namely $P_{E, u}\left(\hat{\mathcal{U}}_{g, p_{s}}^{u}\right)$ is compared to that of its corresponding target vector $\hat{\mathcal{W}}_{g, p_{s}}^{u}$. As seen in Fig. 3, if the trial vector has lower or equal $\mathrm{CF}$ value in comparison to the corresponding target vector, then the trial vector will replace the target vector and it is allowed to proceed to the next generation. Otherwise, the target vector will remain in the population for the next generation. More specifically, the selection procedure may be described mathematically as

$$
\hat{\mathcal{W}}_{g+1, p_{s}}^{u}= \begin{cases}\hat{\mathcal{U}}_{g, p_{s}}^{u}, & P_{E, u}\left(\hat{\mathcal{U}}_{g, p_{s}}^{u}\right) \leq P_{E, u}\left(\hat{\mathcal{W}}_{g, p_{s}}^{u}\right), \\ \hat{\mathcal{W}}_{g, p_{s}}^{u}, & \text { otherwise. }\end{cases}
$$

5) Termination. The ultimate stopping criterion would be that of confirming that the optimal solution of the MSER-MUD has been found. However, we cannot glean any proof of evidence that the MSER-MUD solution has indeed been found. Hence, we opted for halting the optimization procedure, when any of the following stopping criteria are met:

- The pre-defined maximum affordable number of generations $G_{\max }$ has been exhausted.

- $\Delta g_{\max }$ generations have been explored without a trial vector being accepted, which also implies that $\Delta g_{\max }$ generations have passed without any reduction of the CF.

A low-complexity termination criterion is constituted by the number of $\mathrm{CF}$ Evaluations (CF-Evals.), which may be readily used for evaluating the computational complexity imposed. For a given population size $P_{s}$ terminated after $G$ generations, the number of $C F$ Evals. employed for finding the weight vectors $\hat{\mathbf{W}}_{M S E R}^{u}$ representing the MSER solution is equal to $\left(P_{s} \times G\right)$. Hence the total $C F-E v a l s$. for a $U$-user scenario of the proposed DE assisted MSER-MUD is equal to $U\left(P_{s} \times G\right)$. By contrast, the number of CF-Evals. of the optimum ML detector using exhaustive search is equivalent to $M^{U}$. As to the MMSE-MUD [1], there is a closed-form solution, where the computational complexity is dominated by the operation of matrix inversion, namely by a $(Q \times Q)$-element matrix inversion for OFDM/SDMA systems equipped with $Q$ antennas at the BS. It is worth noting that the weight vectors $\hat{\mathbf{W}}_{M S E R}$ of the MSER-MUD solutions acquired may be employed for prolonged time-intervals in the scenarios, when the channels are block-fading or slowly fading. Hence the proposed DE assisted MSER-MUD is more attractive for block-fading or slowly fading scenarios.

\section{Simulation Results}

In this section, our simulation results are presented in order to illustrate the attainable performance of the proposed DE aided MSER-MUD in the context of multi-user OFDM/SDMA systems. It was assumed that the UL multi-user OFDM/SDMA system equipped with $Q=4$ antennas at the BS supported $U=4$ MSs simultaneously transmitting their data in the UL to the BS. A summary of the various parameters used in our simulations is provided in Table I.

TABLE I

Basic Simulation Parameters Used in OUR Simulations

\begin{tabular}{||c|l|l||}
\hline \hline \multirow{3}{*}{\begin{tabular}{c|l} 
FEC encoder \\
and decoder
\end{tabular}} & Type & LDPC \\
& Code rate & $1 / 2$ \\
& Code-word length & 1152 \\
& Maximum No. of iterations & 50 \\
\hline \multirow{5}{*}{ Channel } & Number of paths $L$ & 4 \\
& Delay & $0,1, \cdots, 3$ \\
& Average path gains & {$[0 ;-5 ;-10 ;-15](d B)$} \\
& MSs $U$ & 4 \\
& Received antennas $P$ & 4 \\
& Subcarriers $K$ & 64 \\
& Cyclic prefix $K_{c p}$ & 16 \\
\hline \hline
\end{tabular}

Recall from Section IV that there are three basic algorithmic parameters, namely the population size $P_{s}$, the scaling factor $\lambda$ and the crossover probability $C_{r}$, which influence the performance of the DE assisted MSER-MUD. The number of iterations required for convergence is influenced by the scaling factor $\lambda$ and the crossover probability $C_{r}$, as illustrated by our simulations. The effects of the population size both on the attainable the convergence rate and on the overall BER performance were also characterized.

Specifically, the effects of the scaling factor and the crossover probability on the proposed DE assisted MSER-MUD were investigated in Fig. 4. Observe in Fig. 4 that the proposed scheme required an increasing number of generations for satisfying the termination criterion defined in Section $I V$, when increasing the value of $\lambda$ across the range of $0.4 \leq \lambda \leq 0.9$ and using the fixed crossover probability of $C_{r}=0.6$. Moreover, we can see from Fig. 4 that the number of generations required for achieving convergence monotonically decreased upon increasing the crossover probabilities $C_{r}$, while keeping $\lambda$ in the range of $0.1 \leq \lambda \leq 0.7$. By contrast, the number of generations required for attaining convergence remained nearconstant across the range of $0.8 \leq \lambda \leq 1.0$. The lowest-complexity operating point was found to be at $\left(C_{r}, \lambda\right)=(1.0,0.1)$, yielding $(50 \times 42)=2100 C F$-Evals. However, observe in Fig. 5 that the BER performance of this operating point was suboptimum, which is due to convergence to a local - rather than global - minimum without thoroughly exploring the entire solution space. The BER performance of the operating point $\left(C_{r}, \lambda\right)=(0.1,0.1)$ was optimal, but it imposed an increased complexity, as seen from Fig. 4.

The convergence rate of the proposed DE-aided MSER-MUD at $E_{b} / N_{0}=10 d B, 20 d B$ was characterized in Fig. 6. We can see from Fig. 6 that as expected, the BER was reduced upon increasing the affordable complexity. The proposed scheme exhibits a BER floor at $E_{b} / N_{0}=10 \mathrm{~dB}$, which was eliminated at $E_{b} / N_{0}=20 \mathrm{~dB}$. Furthermore, it can be seen from Fig. 6 that the proposed DE-aided MSER-MUD performs best for $P_{s}=60$, when imposing about $750 C F$-Evals. . Here the complexity imposed was lower than the above-mentioned 'lowest-complexity', since the termination criterion defined in Section IV was a cautious stopping criterion. Indeed, the BER performance may benefit from no substantial improvement before the DE-aided MSER-MUD's evolutionary search was terminated, especially at high values of $E_{b} / N_{0}$.

In Fig. 7 we characterized the overall BER performance of the proposed DE assisted MSER-MUD for a fixed number of CF-Evals., but differently apportioning the total computational complexity of 


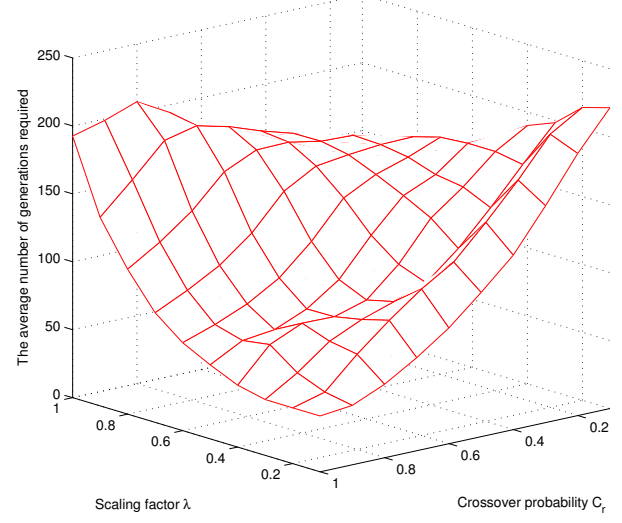

Fig. 4. Average number of evolution generations required for convergence of the proposed DE aided MSER-MUD scheme versus the scaling factor $\lambda$ and crossover probability $C_{r}$ at $E_{b} / N_{0}=12 d B$. All other parameters are given $i$

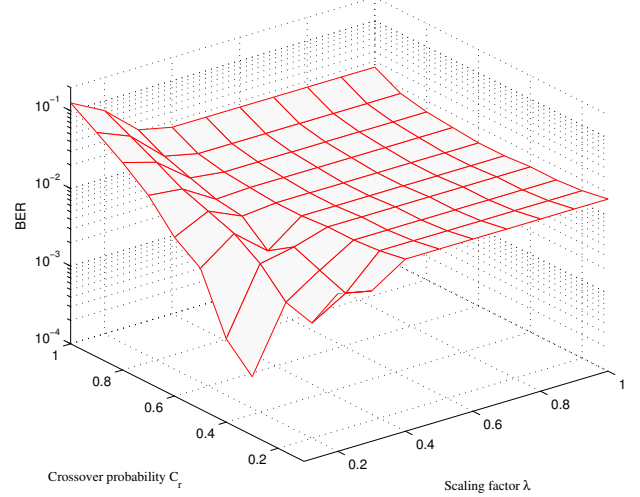

Fig. 5. BER performance of the proposed DE aided MSER-MUD scheme versus the scaling factor $\lambda$ and crossover probability $C_{r}$ at $E_{b} / N_{0}=12 d B$. The number of evolution generations for different combination of $\left(C_{r}, \lambda\right)$ are illustrated in Fig. 4. All other parameters are given in Table I.

$\left(P_{s} \times G\right)=6000$ by varying $P_{s}$ and $G$. The conventional MMSEMUD $[1,3]$ and the optimal ML-MUD were also included as benchmarkers. The MUD weight vectors $\mathbf{W}_{M M S E}$ of the MMSE-based and $\mathbf{W}_{M S E R}$ of the MSER-based solutions were calculated from the pilot symbols and they were then used for the subsequent $N_{s}=72$ OFDM symbols, namely until the next training symbols became available. It can be seen from Fig. 7 that the DE-aided MSERMUD has achieved a significant BER versus $E_{b} / N_{0}$ improvement over the MMSE-MUD, except for the combination of $P_{s}=100$ and $G=60$. But, regrettably, there was still an approximately $4 d B$ performance degradation for the proposed DE-aided MSER-MUD compared to the optimal ML-MUD, albeit, the number of CF-Evals. was only $\frac{U P_{s} G K}{K N_{s} M^{U}}=\frac{4 \times 6000 \times 64}{64 \times 72 \times 16^{4}}=0.51 \%$ for the proposed DEaided MSER-MUD compared to the ML-MUD, which corresponded to an approximately 200 -fold improvement.

\section{Conclusions}

A DE-aided MSER-MUD scheme was proposed for multi-user OFDM/SDMA systems employing $M$-QAM, which directly minimized the SER CF. The proposed MSER-MUD was assisted by the DE algorithm in finding the minimum of the SER CF. The attainable performance versus complexity behaviour was predominately determined by the DE operations of mutation, crossover and selection. The influence of these algorithmic parameters on the proposed DEaided MSER-MUD was investigated using Monte Carlo simulations. It was demonstrated that the proposed DE-aided MSER-MUD is capable of achieving significant performance gains in terms of the system's overall BER over the classic MMSE-MUD and approached the performance of the optimum ML-MUD within about $4 d B$ at about $0.51 \%$ of its complexity.

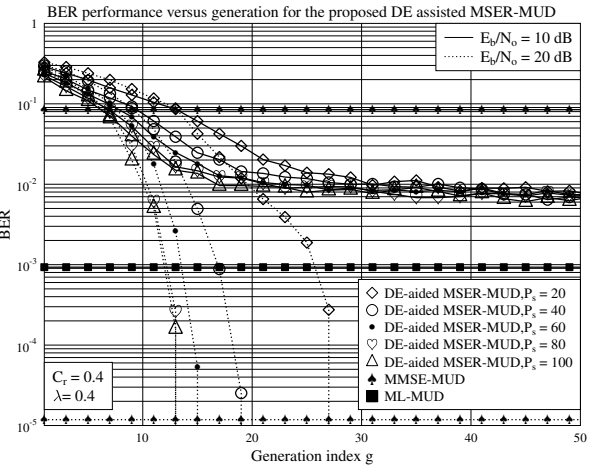

Fig. 6. BER performance against the number of generations required for the proposed DE assisted MSER-MUD scheme. The optimal ML-MUD attains an infinitesimally low BER at $E_{b} / N_{0}=20 \mathrm{~dB}$, which is not shown in this figure. All other parameters are given in Table $\mathrm{I}$.

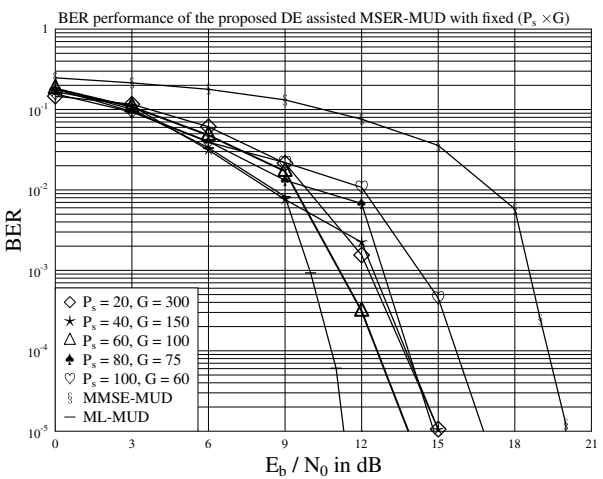

Fig. 7. BER versus $E_{b} / N_{0}$ performance of the LDPC-coded multi-user OFDM/SDMA system. All other parameters are given in Table I.

\section{REFERENCES}

[1] M. Jiang and L. Hanzo, "Multiuser MIMO-OFDM for next-generation wireless systems," Proceedings of the IEEE, vol. 95, no. 7, pp. 14301469, 2007.

[2] L. Hanzo, Y. Akhtman, L. Wang, and M. Jiang, MIMO-OFDM for LTE WIFI and WIMAX: Coherent versus Non-Coherent and Cooperative Turbo-Transceivers. Wiley, 2010.

[3] P. Vandenameele, L. Van Der Perre, M. Engels, B. Gyselinckx, and H. De Man, "A combined OFDM/SDMA approach," IEEE Journal on Selected Areas in Communications, vol. 18, no. 11, pp. 2312-2321, 2000.

[4] S. Thoen, L. Deneire, L. Van der Perre, M. Engels, and H. De Man, "Constrained least squares detector for OFDM/SDMA-based wireless networks," IEEE Transactions on Wireless Communications, vol. 2, no. 1 , pp. 129-140, 2003.

[5] M. Alias, S. Chen, and L. Hanzo, "Multiple-antenna-aided OFDM employing genetic-algorithm-assisted minimum bit error rate multiuser detection," IEEE Transactions on Vehicular Technology, vol. 54, no. 5, pp. 1713-1721, 2005

[6] B. Mulgrew and S. Chen, "Adaptive minimum-BER decision feedback equalisers for binary signalling," Signal Processing, vol. 81, no. 7, pp. 1479-1489, 2001

[7] R. de Lamare and R. Sampaio-Neto, "Adaptive MBER decision feedback multiuser receivers in frequency selective fading channels," IEEE Communications Letters, vol. 7, no. 2, pp. 73-75, 2003.

[8] S. Chen, A. Livingstone, and L. Hanzo, "Minimum bit-error rate design for space-time equalization-based multiuser detection," IEEE Transactions on Communications, vol. 54, no. 5, pp. 824-832, 2006

[9] D. Gesbert, "Robust linear MIMO receivers: A minimum error-rate approach," IEEE Transactions on Signal Processing, vol. 51, no. 11, pp. 2863-2871, 2005.

[10] S. Chen, A. Livingstone, H. Du, and L. Hanzo, "Adaptive minimum symbol error rate beamforming assisted detection for quadrature amplitude modulation," IEEE Transactions on Wireless Communications, plitude modulation," IEEE Transaction
vol. 7, no. 4, pp. 1140-1145, 2008.

[11] K. Price, R. Storn, and J. Lampinen, Differential evolution: a practical approach to global optimization. Springer Verlag, 2005.

[12] A. Qin, V. Huang, and P. Suganthan, "Differential evolution algorithm with strategy adaptation for global numerical optimization," IEEE Transactions on Evolutionary Computation, vol. 13, no. 2, pp. 398-417, 2009.

[13] J. Proakis and M. Salehi, Digital communications. McGraw-hill Companies, Inc., New York, 2001.

[14] S. Winitzki, "A handy approximation for the error function and its inverse," A lecture note obtained through private communication, 2008. 